\title{
Neue Versuche über die Vita propria.
}

\author{
(Nachtrag zu der Arbeit: \\ Über Transplantation zusammengesetzter Theile 1) s.) \\ Von \\ S. Saltykow, \\ Assistent am pathologischen Institut in Groningen. \\ Nit 'Tafel XVIII. \\ Eingegangen am 26. Juni 1901.
}

In meiner früheren Arbeit ${ }^{1}$ ) bin ich zum Schluss gekommen, dass es sich bei Transplantation stets um mehr oder weniger ausgesprochenen Untergang und Wiederherstellung der transplantirten Gewebe handelte.

Unter Anderen hatte ich Versuche mit Rattenschwänzen angestellt, welche vor der Transplantation eine Zeit lang aufbewahrt worden waren (pag. 376).

Da aber die Regeneration in diesen letzten Versuchen bei vorausgegangener 14 täger Aufbewahrung verhältnismäßig wenig ausgesprochen war, so habe ich zur nochmaligen Kontrolle neue Versuche angestellt, und zwar unter möglichst gtinstigen Bedingungen. Als solche wurden damals schon jüngeres Alter der Versuchsthiere und aseptische Aufbewahrung des Transplantationsmaterials bei niedriger Temperatur erwähnt. Es wurden sechs grawweike, meist unter einem Monat alte Ratten gebraucht (nur Ratten 2 und 5 waren ïber einen Monat alt). Die abgeschnittenen Schwänze wurden sämmtlich 14 Tage im Eisschrank in Agarröhreben aufgehoben. Nach der Herausnahme des Schwanzes sind nur in zwei Fällen einzelne

1) Dieses Archiv. 1900. Bd. IX. pag. 329. 
Kolonien auf dem Agar im Brutofen anfgetreten, die ibrigen Röhrchen sind steril geblieben.

Die sonstige Untersuchungstechnik war die nämliche wie in den früheren Versuchen.

Im Weiteren werde ich nur kurze Protokolle der neuen Versuche mittheilen.

Die 14 Tage aufbewahrten Gewebe zeigen vor der Transplantation Verkleinerung, Schrumpfung der Kerne, doch sind verhältnismäßig viele Kerne gut erhalten, besonders in den Chordaresten. Die kontraktile Substanz der Muskeln hat ihre Querstreifung eingebüßt.

Ratte 1. Versuchsdauer (nach der Implantation unter die Rickenhaut) 4 Tage.

Im Knochen-, Sehnen- und Nervengewebe sind die meisten Kerne in Zerfall begriffen. Ein Theil der Kerne ist gut erhalten. Knorpel und Chordarest (Fig. 1) zeigen beträchtliche Zellvermehrung mit Mitosen. In den Bandscheiben finden sich stellenweise neugebildete Spindelzellen, vielfach mit Mitosen. Die Muskelkerne sind stellenweise vermehrt.

Ratte 2. Versuchsdauer 8 Tage.

Die meisten Kerne in den Knochen, im Knorpel und in den Nerven sind verschwunden; nirgends deutliche Wucherung. Die meisten Sehnen sind fast kernlos, stellenweise zeigen sie aber Vermehrung der Kerne. Die Muskeln lassen in bedentender Ausdehnung Wucherung der Kerne und Bildung von Sarkoblasten mit Mitosen erkennen (Fig. 2). Dies ist hauptsächlich in den peripheren Theilen des Schwanzes zu sehen. In den centralen Partien sind meist nur spärliche, geschrumpfte Kerne erhalten.

Ratte 3. Versuchsdauer 8 Tage.

Im Ganzen wenig Wucherung.

Hier und da Vermehrung der Muskelkerne und Sarkoblastenbildung. Ziemlich viele gut erhaltene Sehnenkerne. Wucherung der Chordazellen sieht man nur in der Bandscheibe, welche beim Abtrennen des Schwanzes durchgeschnitten wurde. Nur einzelne erhaltene Kerne finden sich in den Nerven.

Ratte 4. Versuchsdauer 14 Tage.

Im Knochenmark sind zahlreiche Markzellen zwischen den Fettzellen a ufgetreten. Bildung ron jungen 
Fettzellen ist auch zu sehen. In den peripheren Knorpelpartien finden sich größere Inseln von neugebildeten Zellen (Fig. 3). In den Bandscheiben ziemlich zahlreiche neugebildete Spindelzellen. Das Chordagewebe ist zellreich und stellenweise in den Bandscheibenknorpel hineingewuchert. Die Nerven sind sebr kernreich, enthalten stellenweise Spindelzellen. Die Sehnen weisen sehr kernreiche Absehnitte anf. In den Muskeln beobachtet man Kernwucherung an den spärlichen erhaltenen alten Fasern; in das Bindegewebe sind spindelförmige Sarkoblaten eingelagert.

Ratte 5. Versuchsdauer 22 Tage.

Wenig Regeneration. Die Gewebe sind zum größten Theil kernlos. In den Sehnen und Nerven sind nur kleine kernreiche Bezirke sichtbar. In den Bandscheiben inselförmige Nenbildung von Spindelzellen. Auch die Chordazellen sind gruppenweise gewuchert. Die Muskeln zeigen Vermehrnng der Kerne und zerstreute Sarkoblasten im Bindegewebe.

Ratte 6. Versuchsdaner 24 Tage.

Chorda ist zellreich und zeigt knorpelige Umwandlung. Die Bandscheiben enthalten viele Zellen in ihren centralen Partien. Auch die Sehnen und Nerven sind stellenweise ziemlich kernreich. Im Knorpel finden sich beträchtliche Inseln von neugebildeten Zellen. Das Knochenmark ist zellreich und zeigt an den epiphysären Enden viele protoplasmareiche, zum Theil mehrkernige Zellen. Stellenweise hat Ablagerung von neugebildeter Knochensubstanz auf die alten Bälkchen stattgefunden. Muskeln sind mit Sicherheit nicht nachzuweisen.

Wie ans diesen Befunden ersichtlich ist, kann ich nicht nur meine frtheren Beobachtungen bestätigen, sondern ich habe manchmal sogar eine viel stärker ausgesprochene Wucherung nach der Transplantation von 14 Tage lang aufbewahrten Theilen beobachtet als in den früheren Versuchen.

\section{Erklärung der Abbildungen.}

Tafel XVIII.

Fig. 1. Versuch 1. Dauer der Transplantation 4 Tage (nach 14 tägiger Aufbewahrung. Chordarest. Links und unten angrenzende Partien des Bandscheibengewebes. In diesem sieht nur eine Zelle (oben links) wie neuge- 
bildet aus. Die uibrigen Zellen sind nur in Form ron blassen Schatten mit undeutlich kontourirten Kernen erhalten. Möglicher Weise sind einzelne von diesen Zellen noch wucherungsfähig. Im Chordarest sind sämmtliche Zellen neugebildet resp. gewuchert. Links unten eine Mitose. Rechts rier verästelte Schleimklumpen (dunkel gefärbt). Vergr. 400.

Fig. 2. Versuch 2. Dauer 8 Tage. Muskeln. Das untere, rechte Viertel ist vom Sehnengewebe eingenommen. In diesem nur eine Zelle (rechts) gewuchert, sonst nur schmale, blasse Reste von solchen. Die kontraktile Substanz der Muskelfasern ist degenerirt, homogen. Die Kerne sind stark vermehrt, viele von ihnen von einem körnigen Protoplasmahof umgeben. Am oberen Rande der Sehne vier frei liegende Sarkoblasten. Weiter nach rechts hin ein Sarkoblast mit Mitose. In den Muskelfasern und zwischen denselben meist kugelige, kleine, dunkle, zu Grunde gehende alte Muskelkerne zerstreut. Vergr. 400.

Fig. 3. Versuch 4. Dauer 14 Tage. Knorpel. In der mittleren Zone verschieden große, in gemeinschaftliehen Kapseln gelagerte Gruppen ivon neugebildeten Knorpelzellen. Oben und unten leere oder blasse Zellreste einschließende Kapseln. Zwei von solchen Zellresten (in der Mitte und links unten) enthalten gut erhaltene große Kernkörperchen. Vergr. 180. 


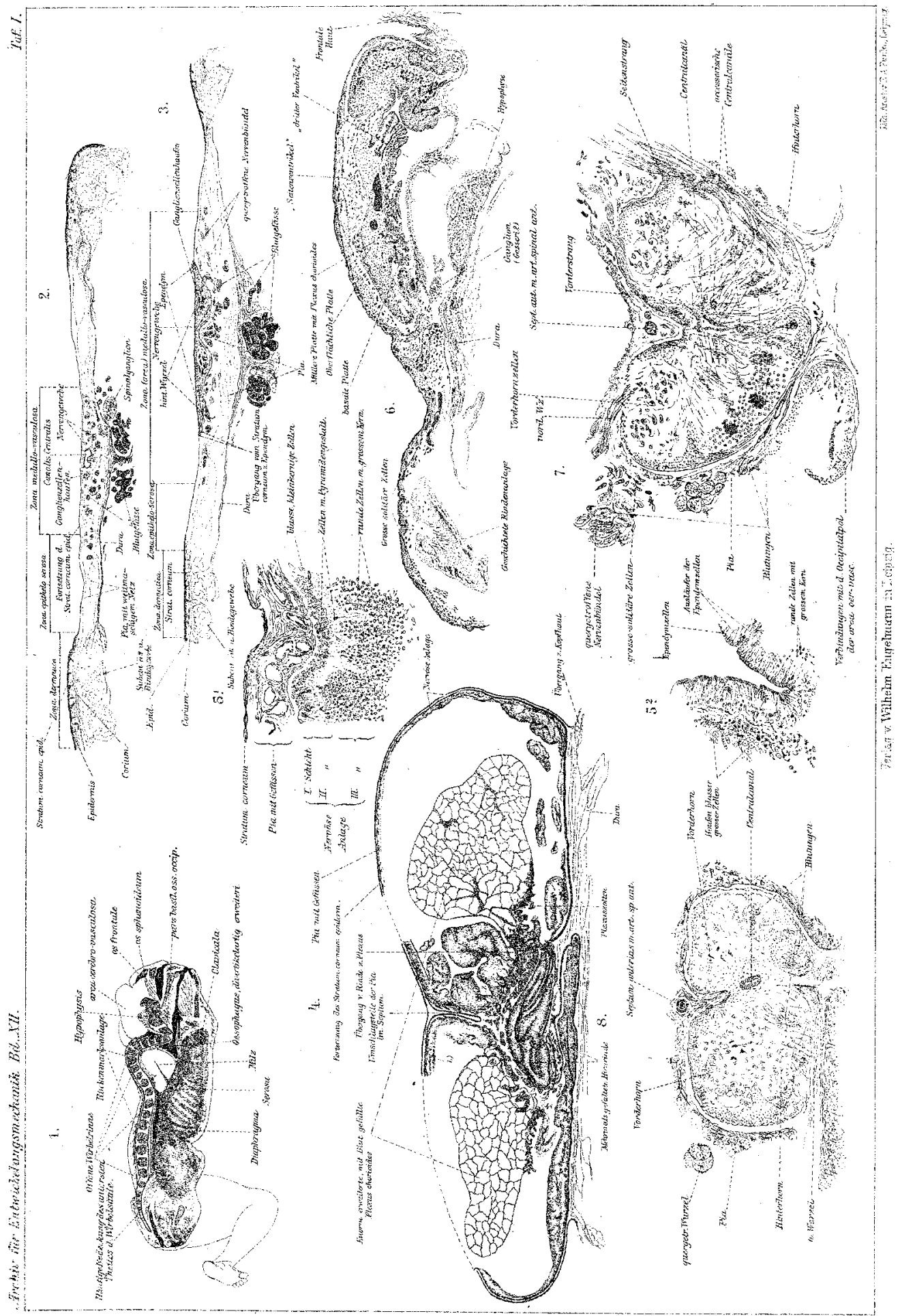




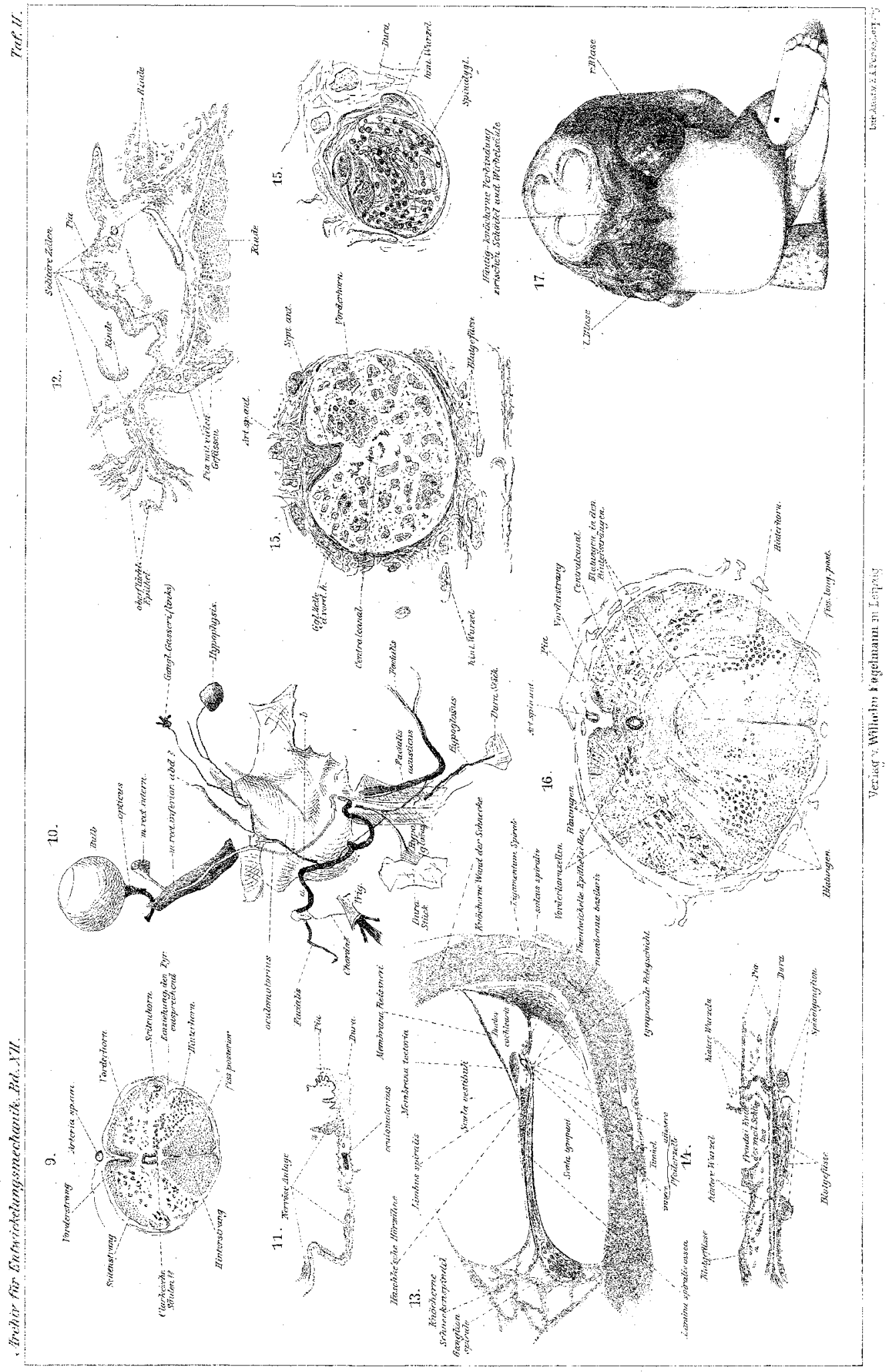




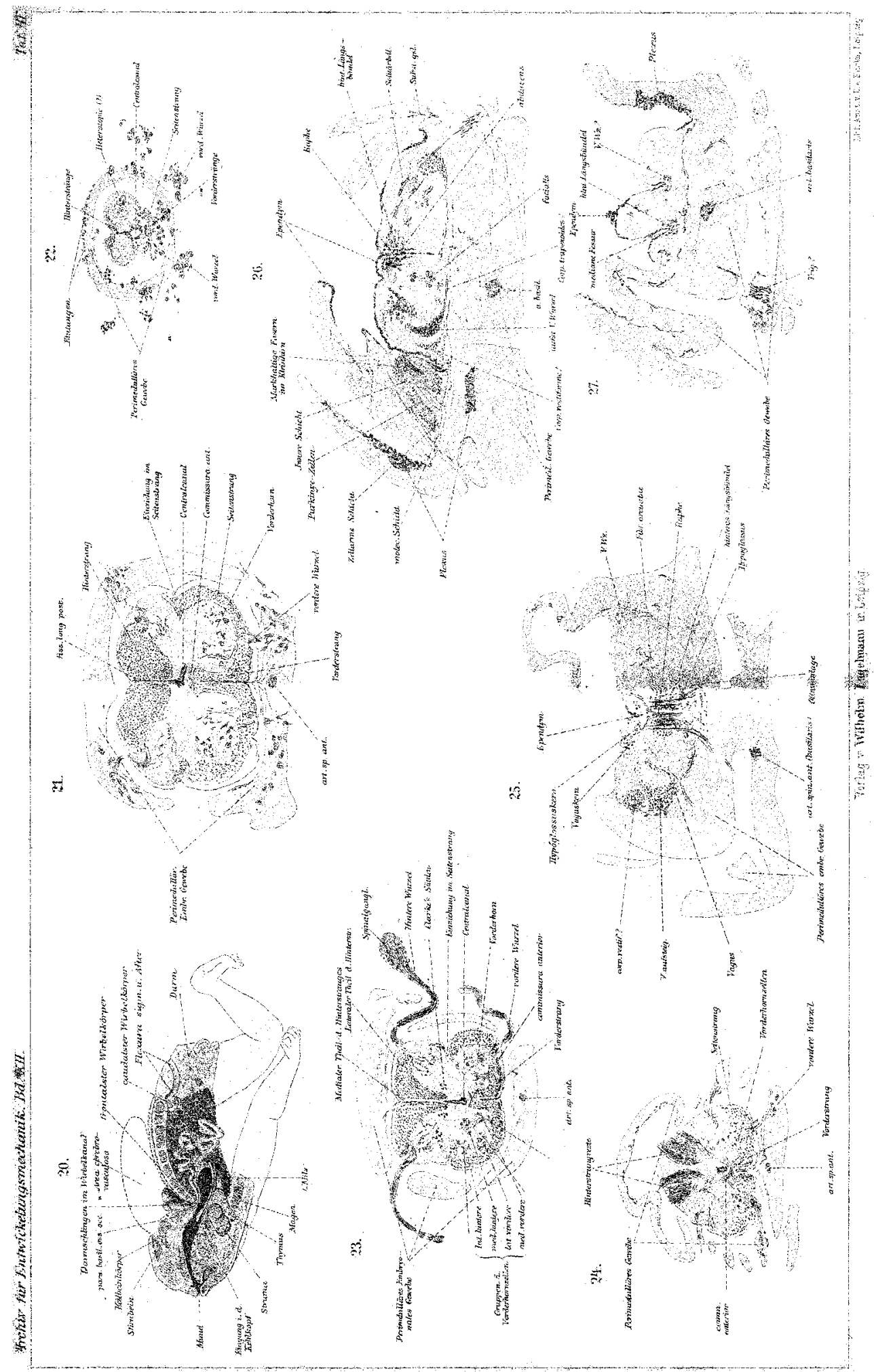




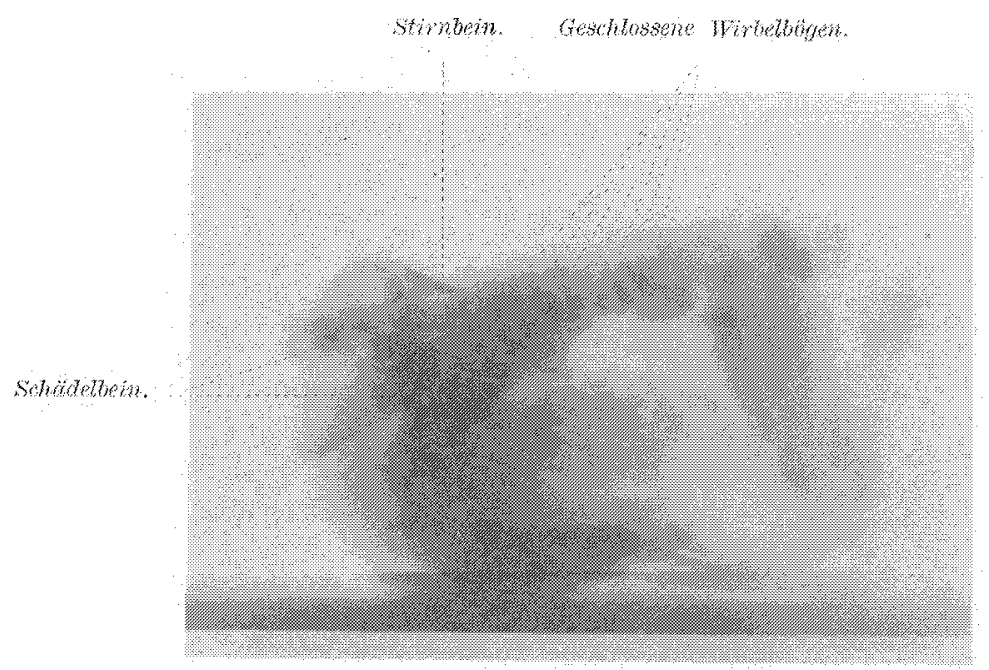

girs. 19. Fall Wh, won der Soite gesehon.

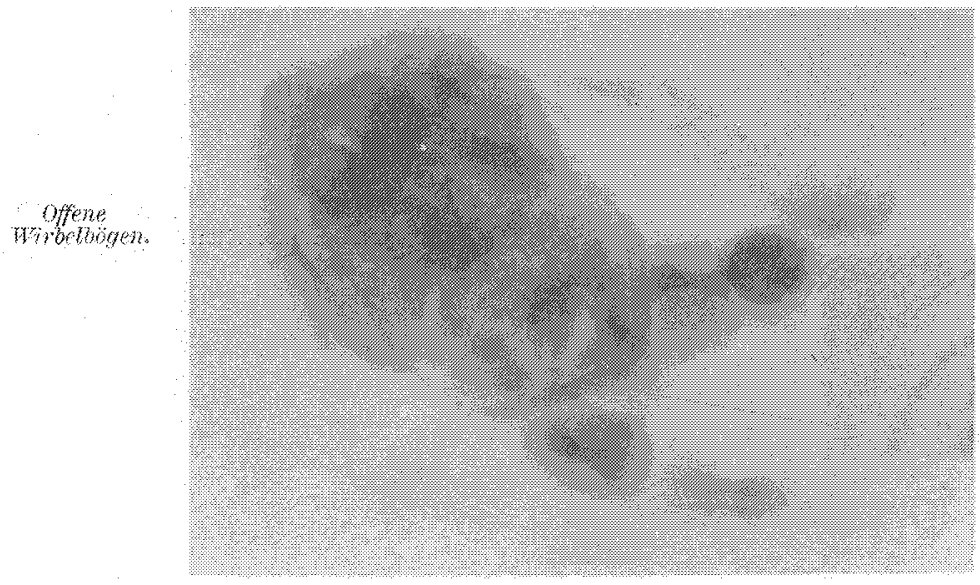

Fis. 19. Fall Wh, wom obu gesehen. 


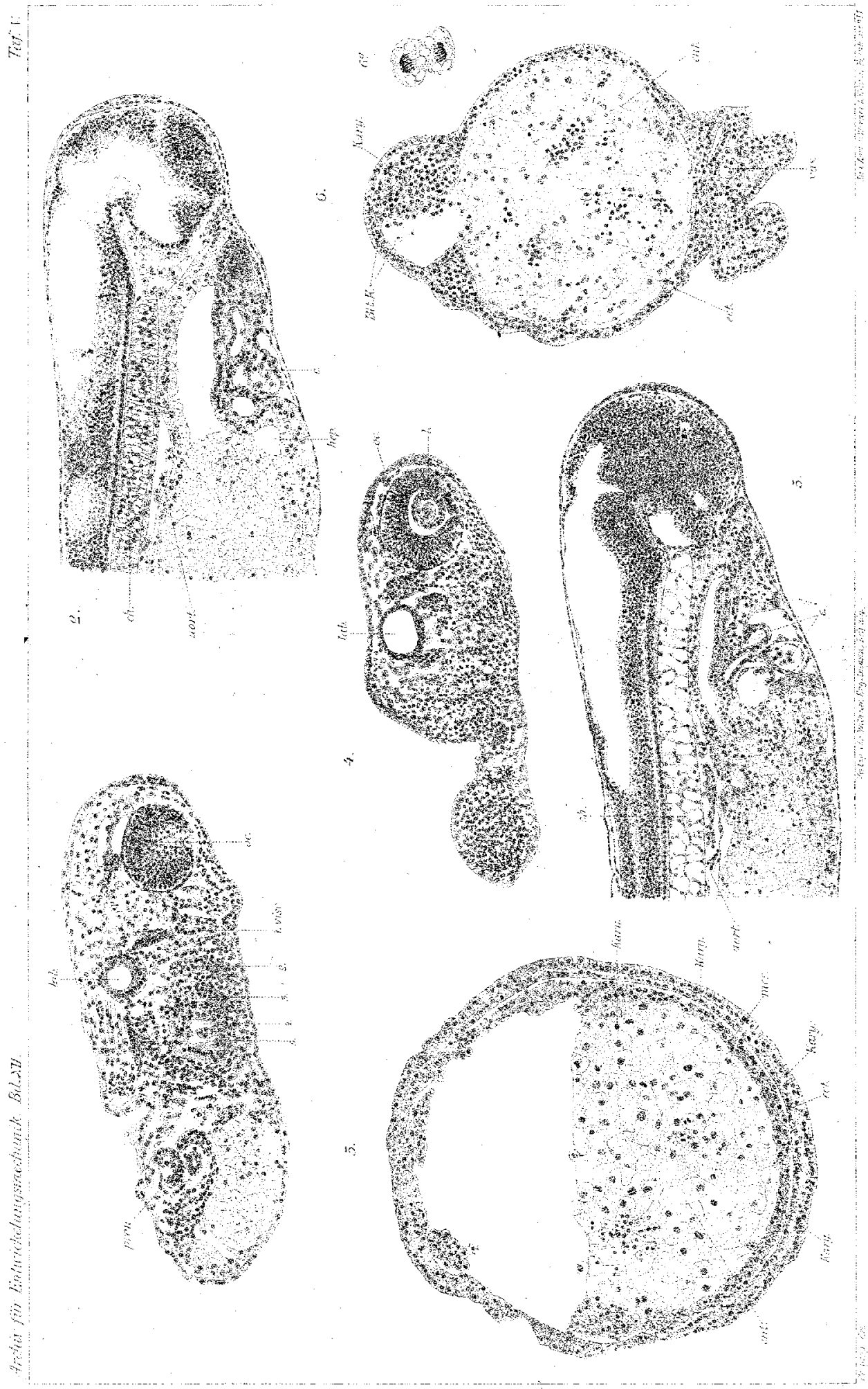




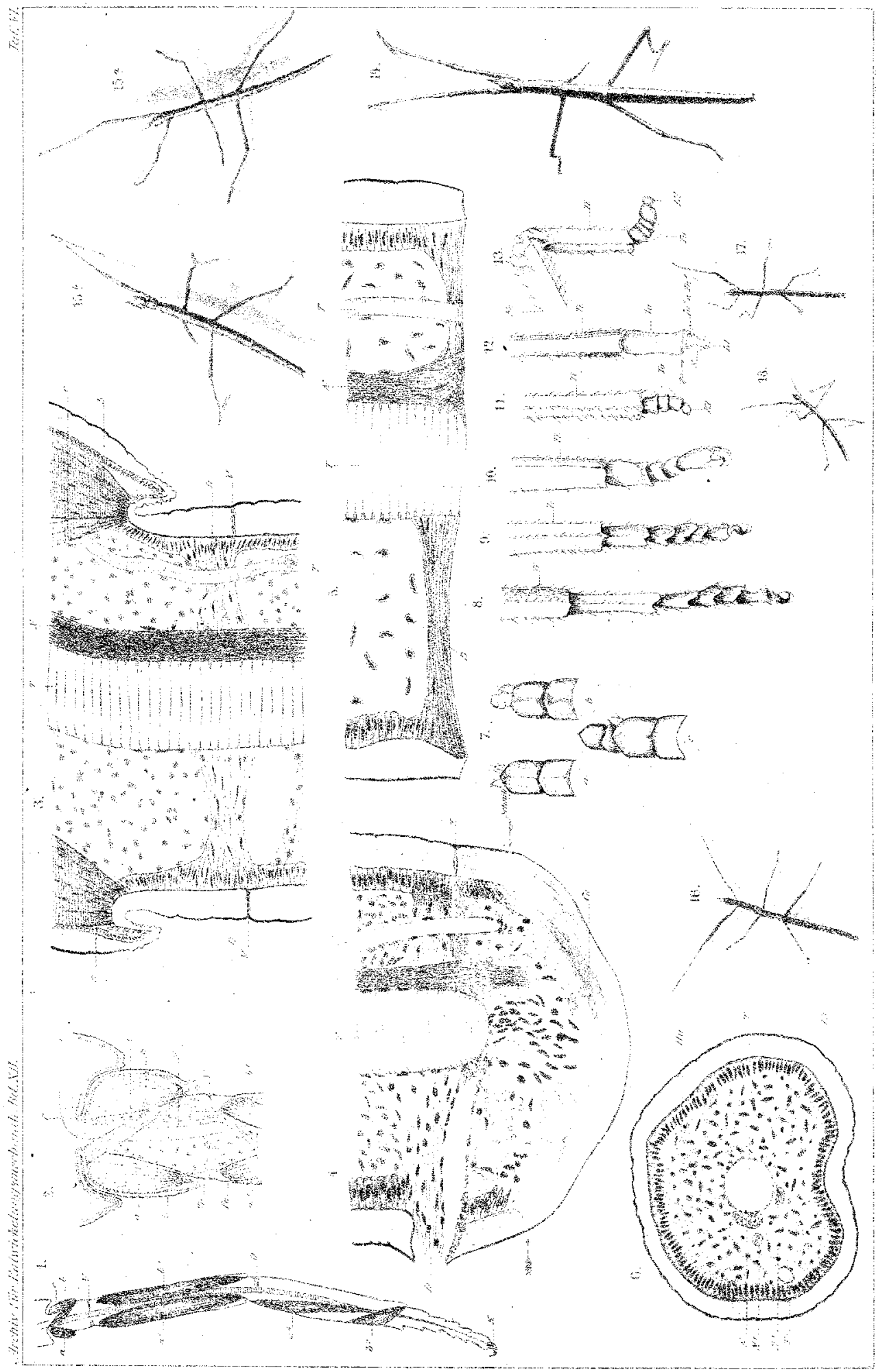




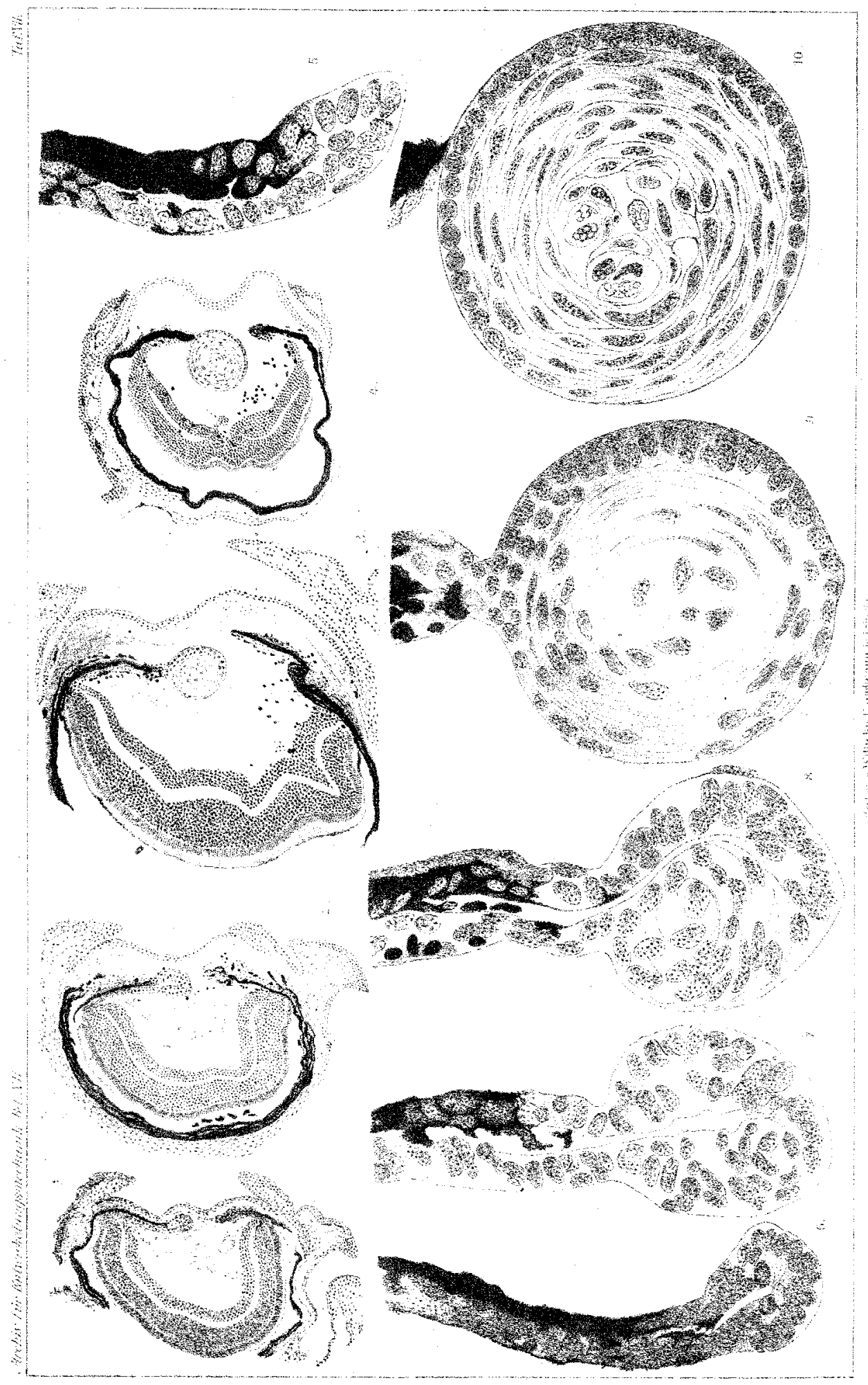




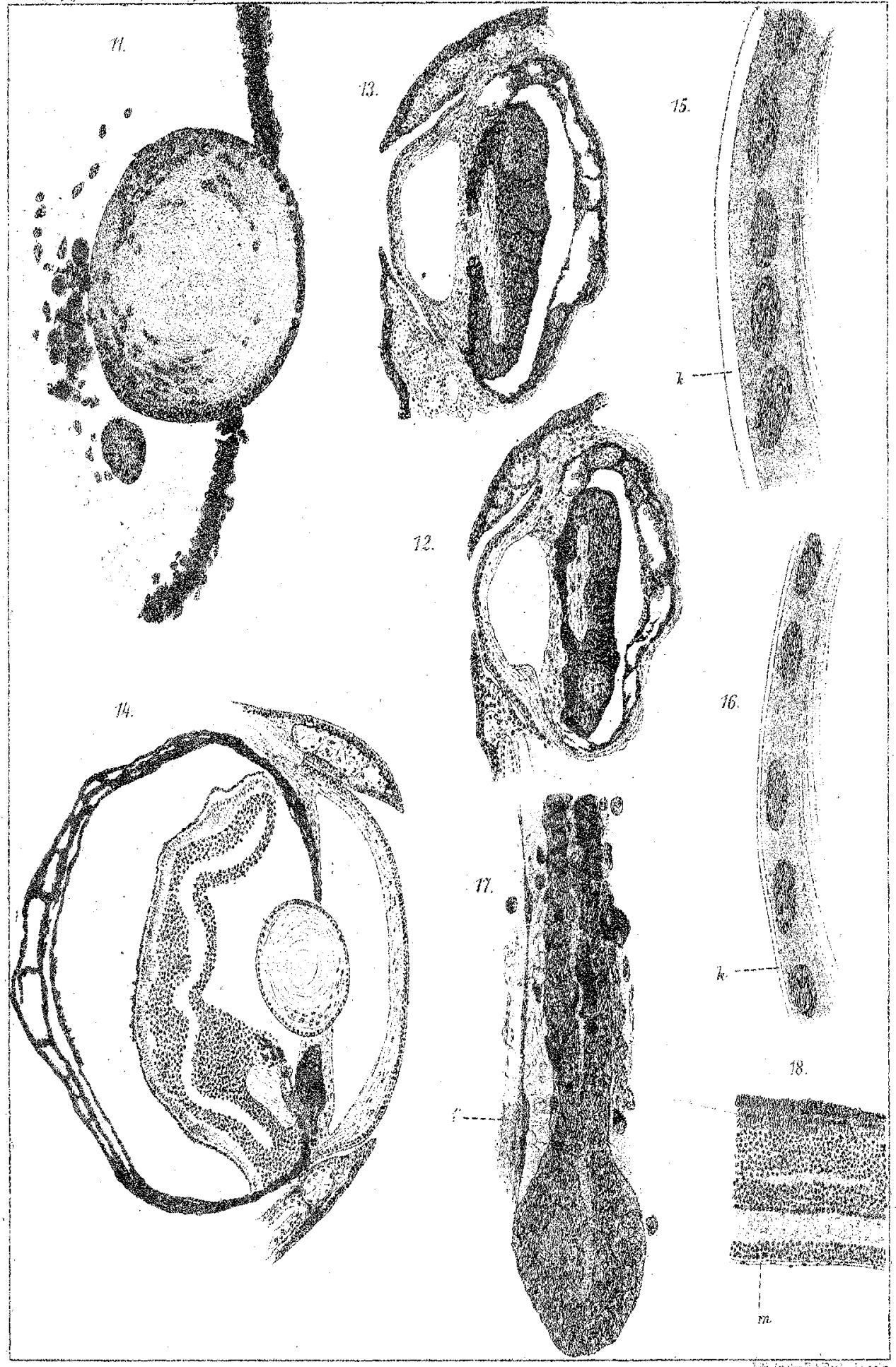



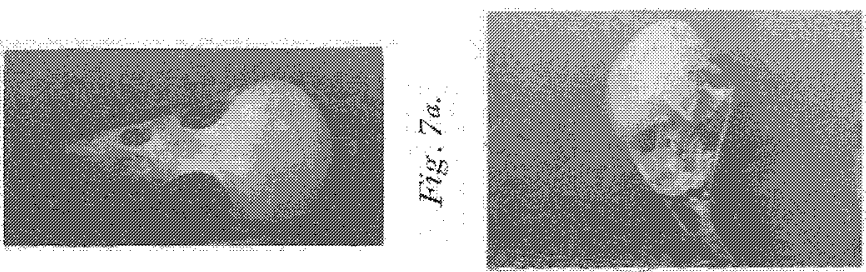

8

$\vdots$
$b$
0
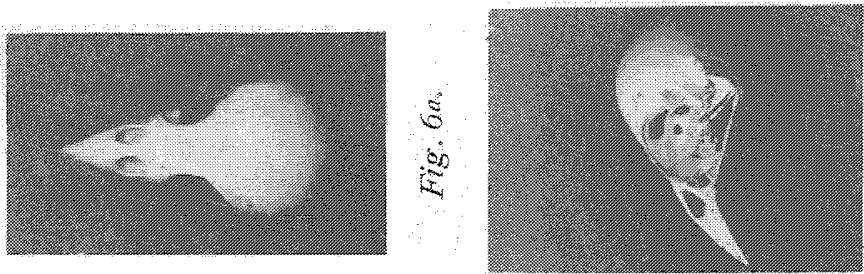

6

is
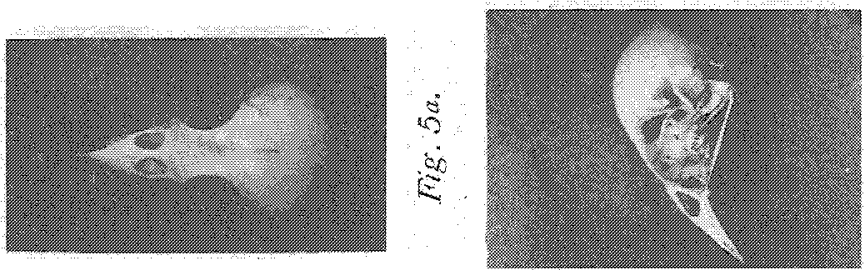

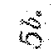

8
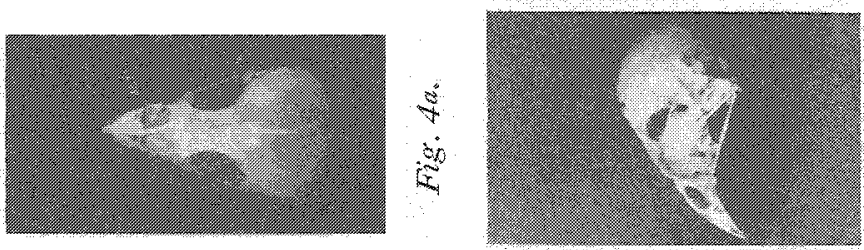

8
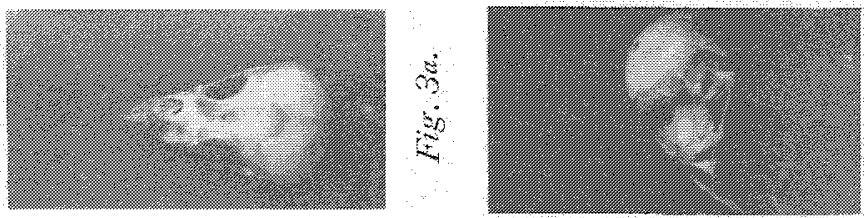

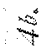

$\infty_{i}^{\infty}$
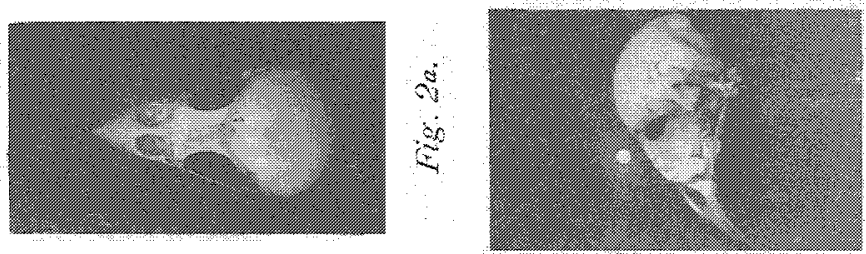

iิ

㱐
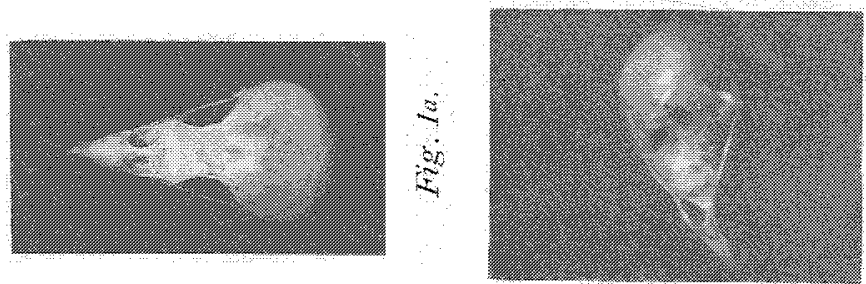

$\approx$

和

$\approx$

8 


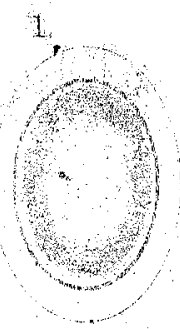

6

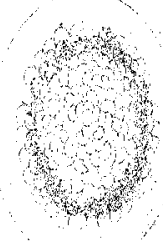

11.

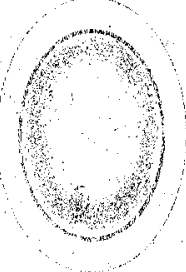

10

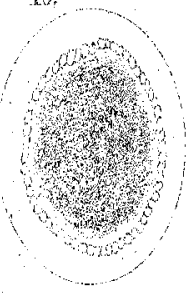

9. it.

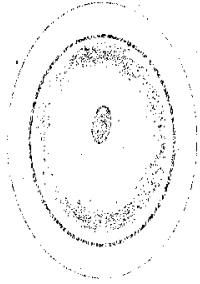

20

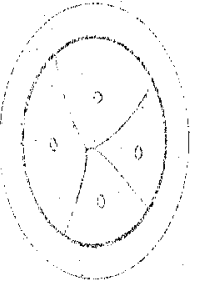

18.
4.

i.

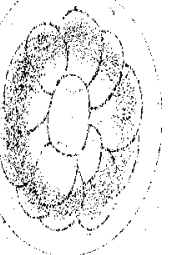

is

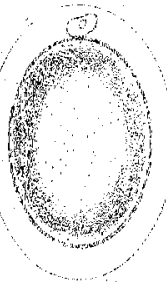

14.
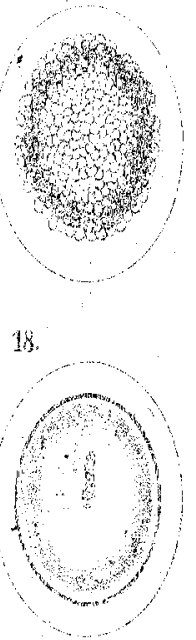

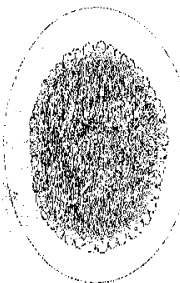

11.

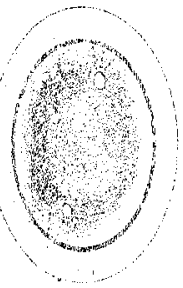

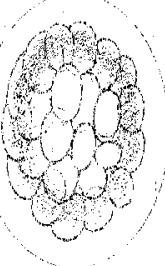

10

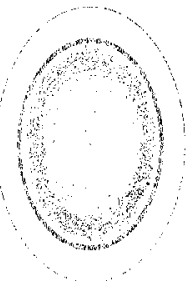

[5.

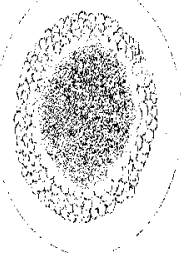

91.

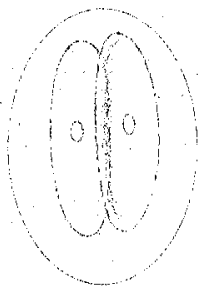

04

;is,
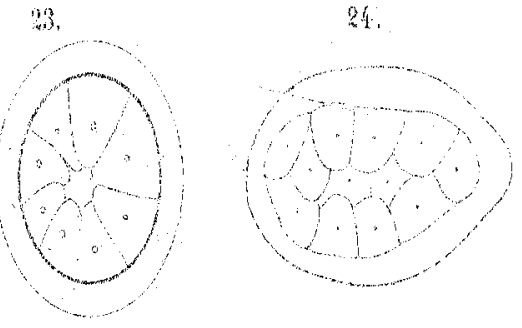

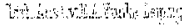




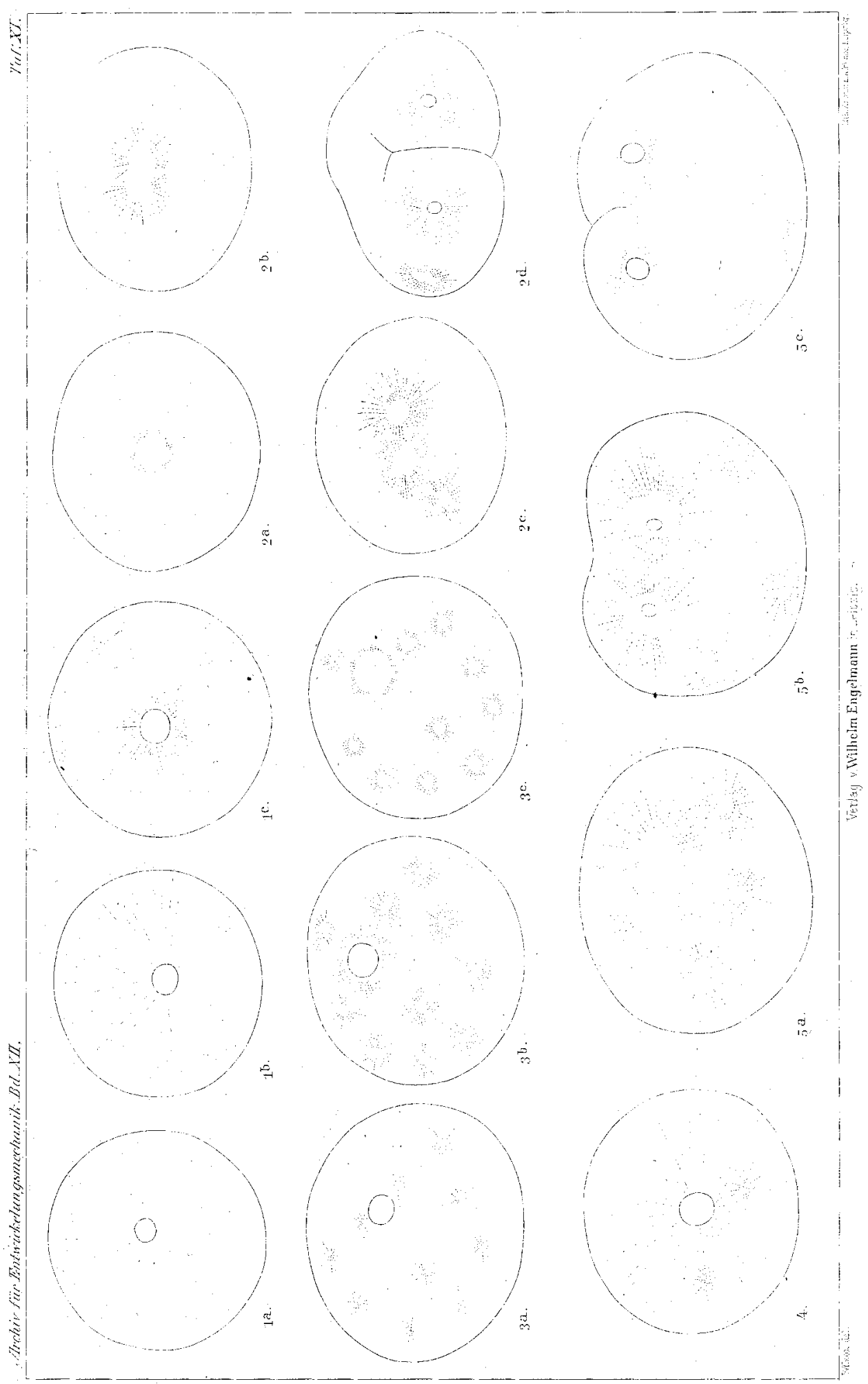




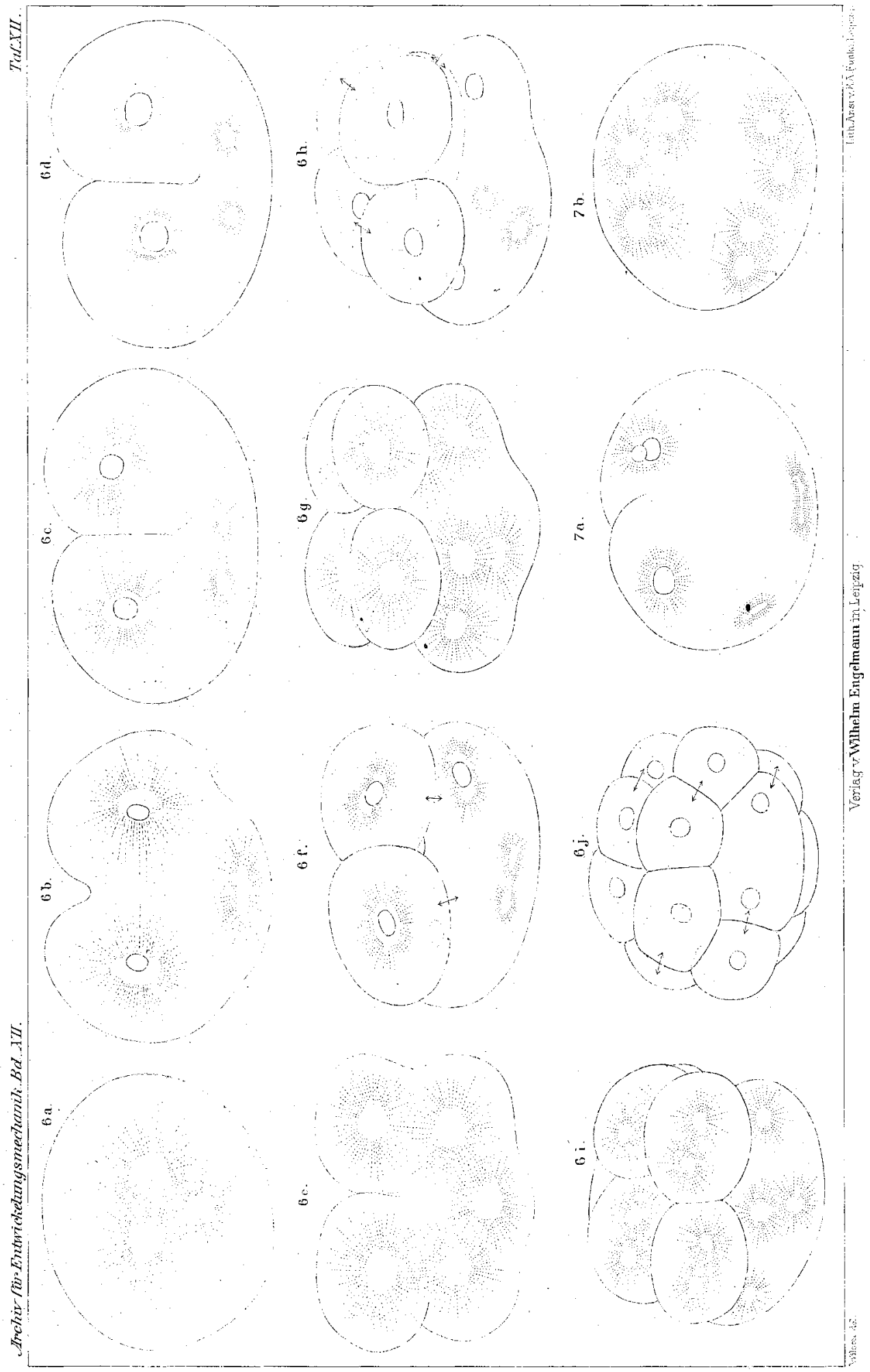




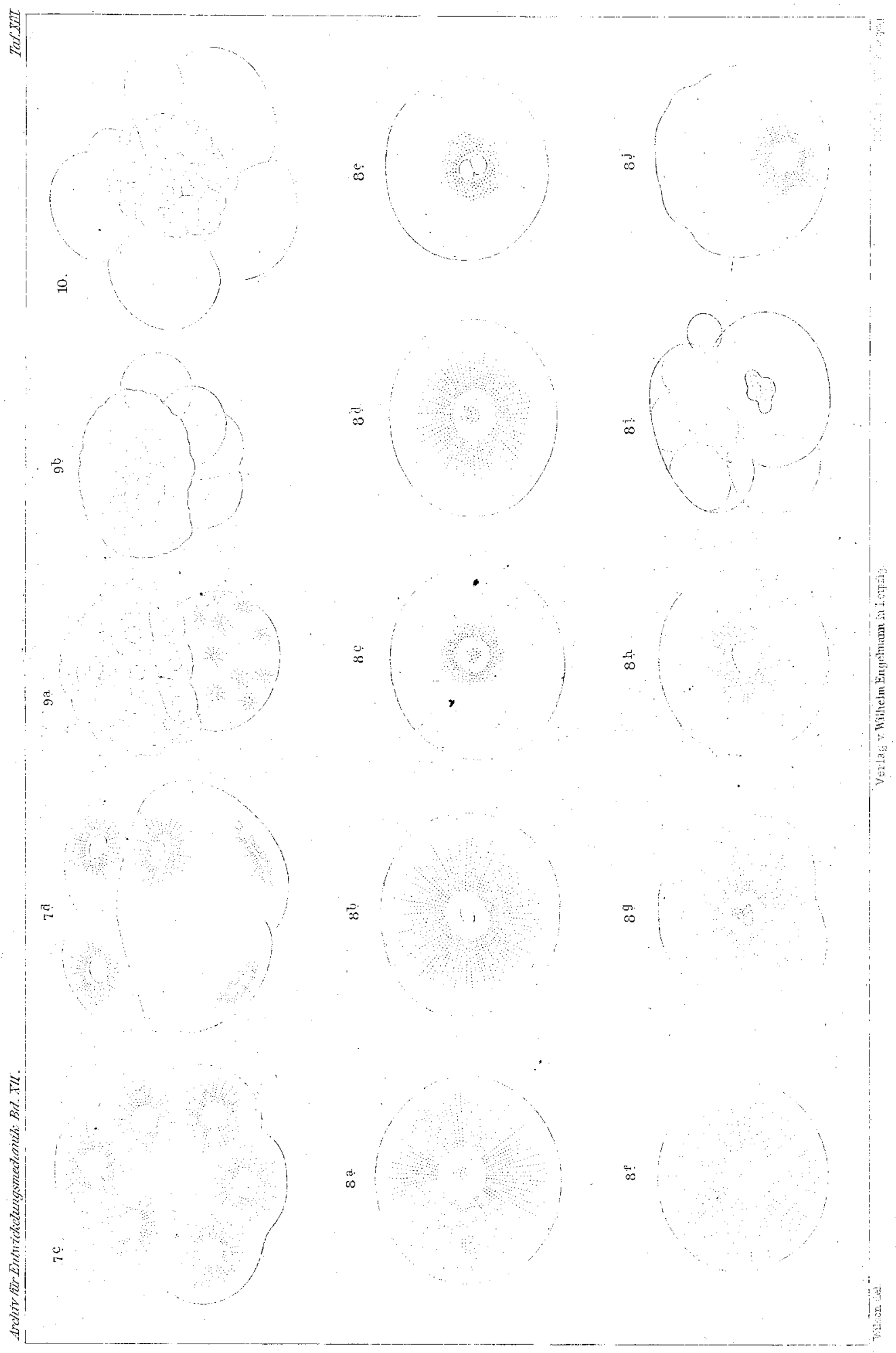




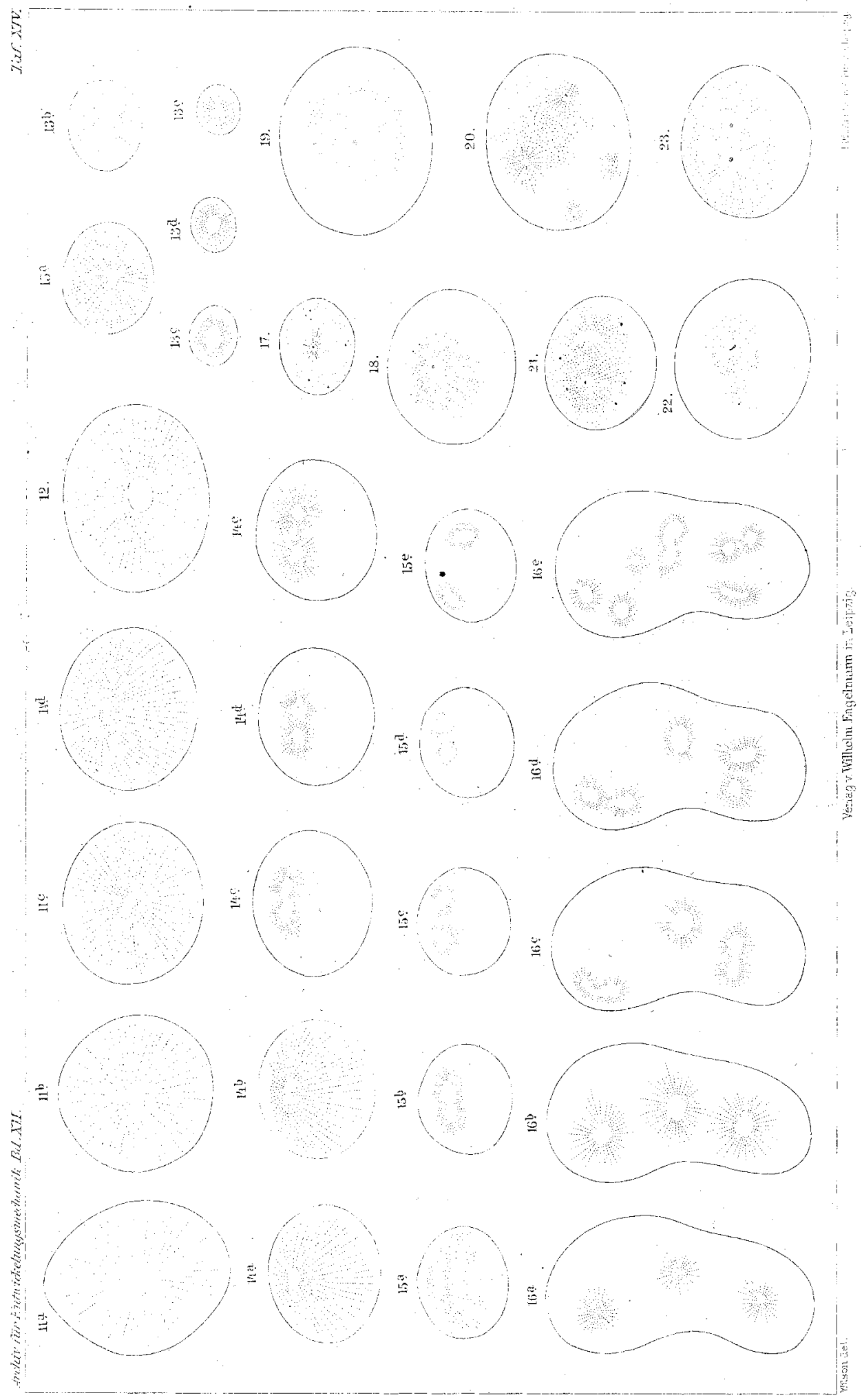




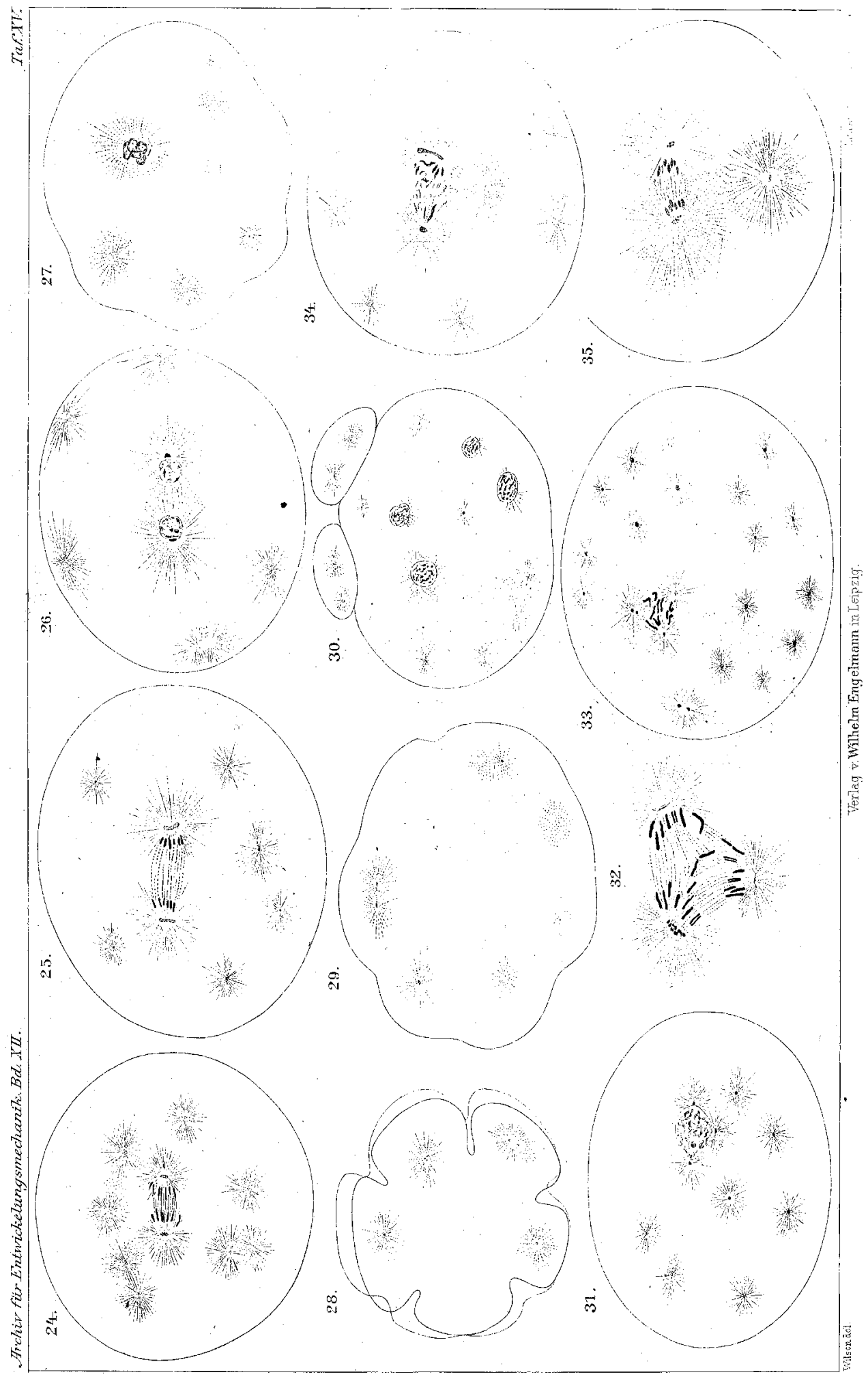




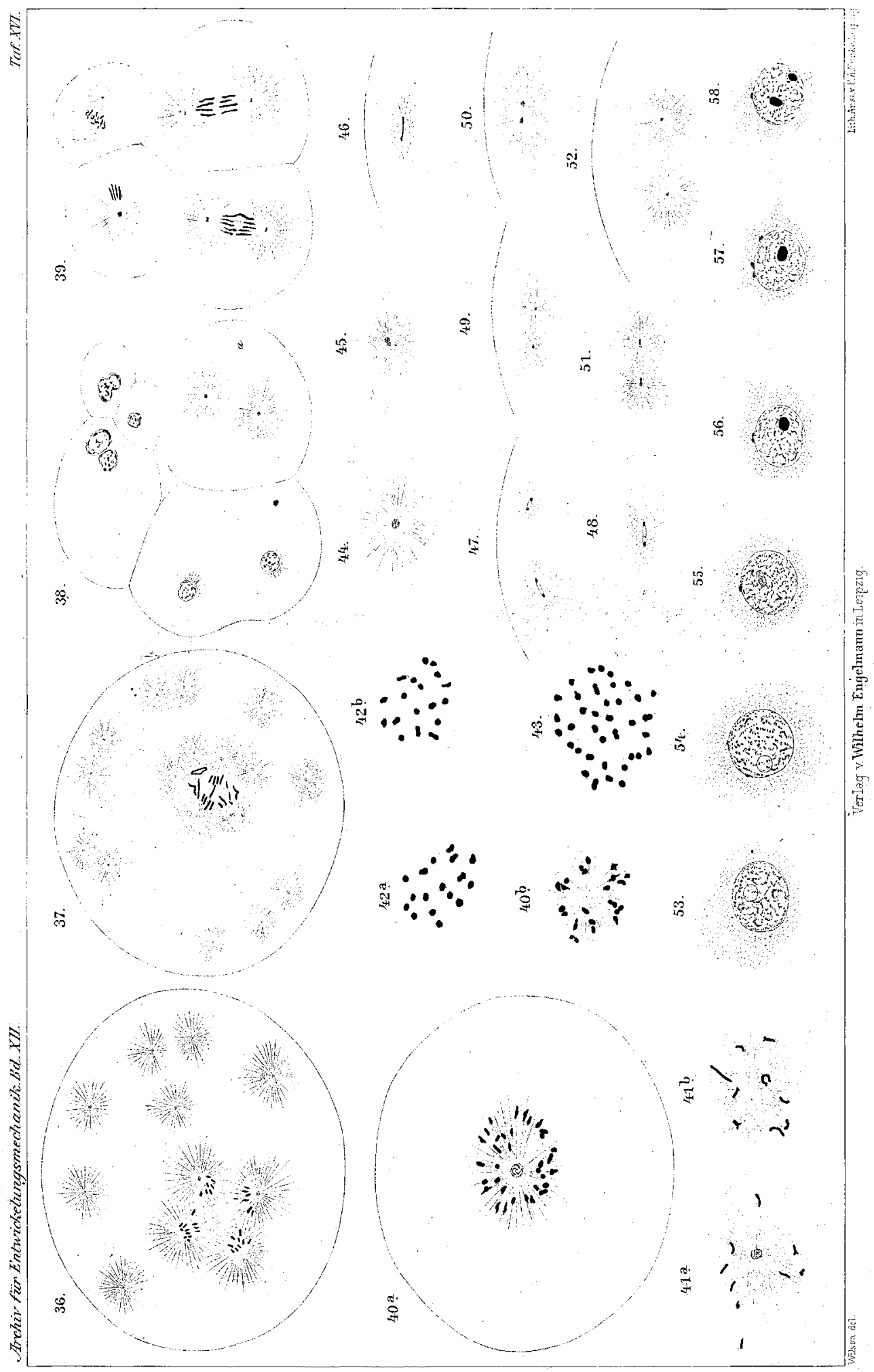


$\frac{2}{3}$

.

$\div \log$

$\sqrt{3}$

क

$\dot{8}$

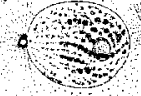

$\mathrm{s}^{-1}$

छ்

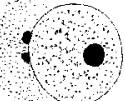

$\div(10)$ का

5

$8+\mathrm{ln}^{2}$

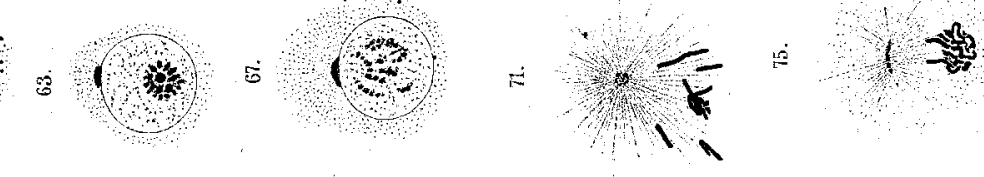

(1)

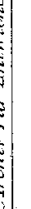

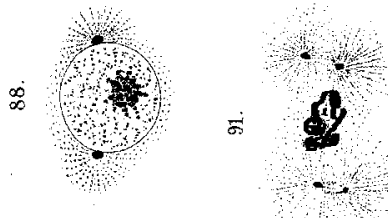

$\infty$

\&

बे

13

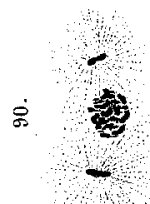




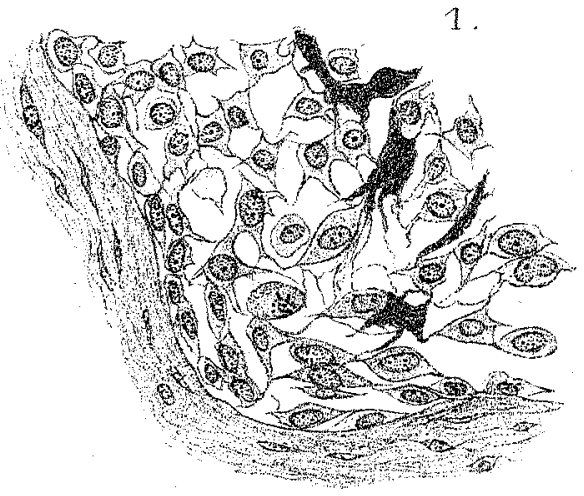

2 .
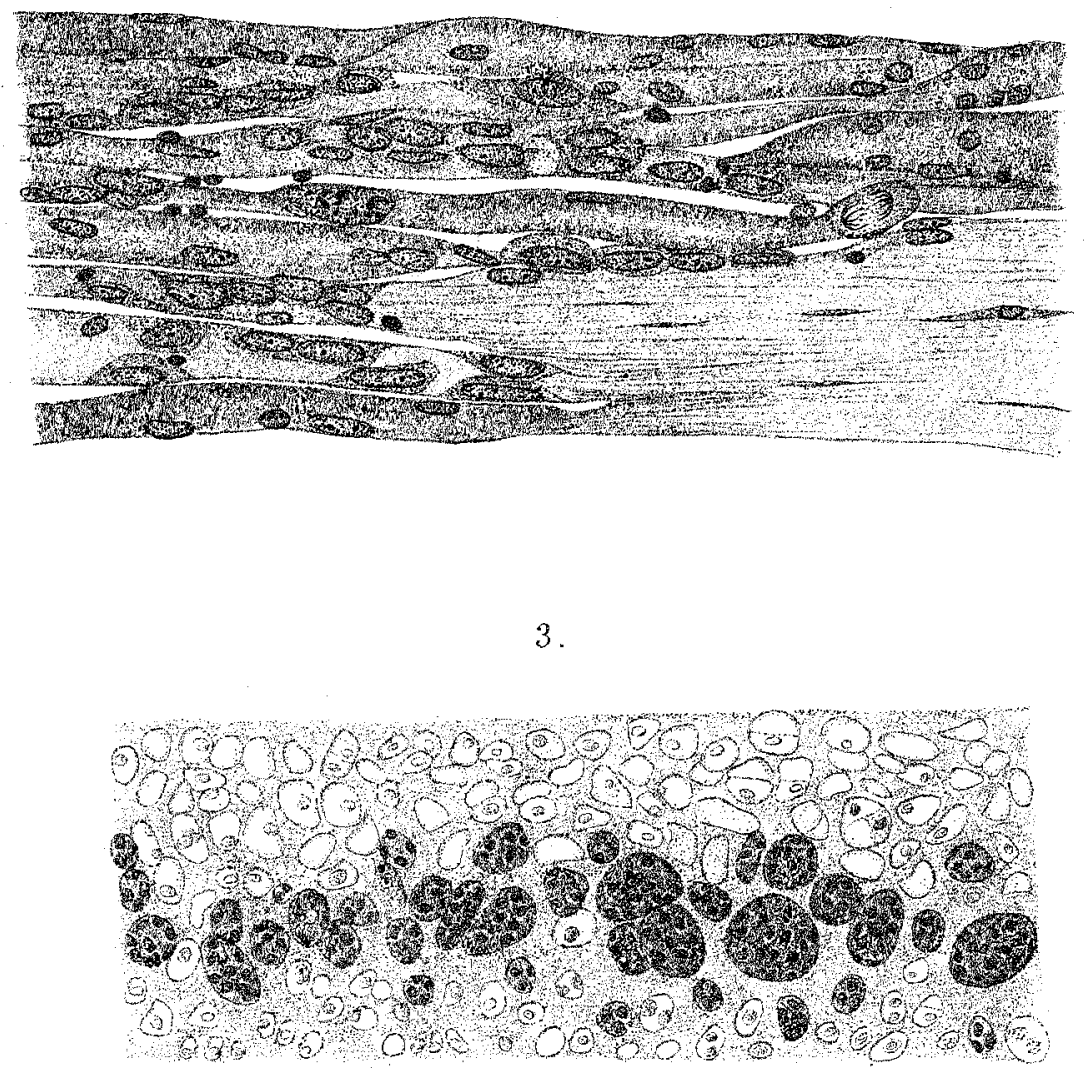